\title{
A Design-of-Experiments Approach to Developing Thermoresponsive Gelators From Complex Polymer Mixtures
}

Received 00th January 20xx, Accepted 00th January 20xx

DOI: $10.1039 / x 0 x \times 00000 x$

\author{
Mohamad A. Abou-Shamat, Jacqueline L. Stair, Stewart B. Kirton, Jesus Calvo-Castro and Michael T. \\ Cook*
}

\begin{abstract}
This study investigated the effects of additives on the properties of poloxamer (P) 407 thermogels, employing a Design-ofExperiments (DoE) approach. P407 is a thermoresponsive triblock copolymer that exhibits a solution to gel transition at a critical temperature, typically between $15-25^{\circ} \mathrm{C}$, dependant on polymer concentration. This thermoresponsive gelation has made P407 attractive for many applications including drug delivery, cell culture and tissue engineering. However, the gels formed do not have sufficient strength for some applications where the materials will be exposed to shear, such as topical drug delivery. There have been attempts to improve P407 thermogel properties by the addition of other hydrophilic polymers. However, these studies were limited to a small number of polymers, typically in binary mixtures, exploring one variable at a time. In this study, a DoE approach was carried out using a two-level model exploring P407, P188, poly(vinyl alcohol), poly(ethylene glycol), and poly(acrylic acid) as variables, including an exploration of molecular weight of the latter three additives. The thermoresponsive gelation of the 16 generated formulations was studied by rheometry and predictive models built for gel strength $\left(G^{\prime}\right)$ and gelation temperature $\left(T_{\text {gel }}\right)$ responses. The model was able to predict the thermoresponsive gelation of complex octonary test blends, significantly streamlining formulation development processes relative to current methods. The model was then able to identify novel thermoresponsive gel formulations with $20 \%$ improved gel strength compared to a standard $20 \%$ P407 solution, which may be used as temperature-responsive materials for advanced healthcare applications.
\end{abstract}

\section{Introduction}

"Thermogels",

"thermoresponsive

gelators"

or "thermoreversible gelators" are aqueous polymer solutions that exhibit a sol-gel transition above a critical temperature. A number of synthetic polymers, ${ }^{1,2}$ peptides, $^{3}$ and polysaccharides ${ }^{4}$ have been investigated as thermoreversible gelators for applications such as drug delivery, cell culture, wound dressing, biofabrication and tissue engineering. ${ }^{5-10}$ Poloxamers (Ps) are a group of thermoresponsive poly(ethylene oxide)-b-poly(propylene glycol)-b-poly(ethylene oxide) (PEOPPO-PEO) amphiphilic triblock copolymers which can be synthesised with various molecular masses and different $\mathrm{PEO} / \mathrm{PPO}$ ratios. For example, $\mathrm{P} 184$ consists of $\mathrm{P}(\mathrm{EO})_{13}-\mathrm{b}-$ $P(P O)_{30}-b-P(E O)_{13}$ and an average molecular mass of $2900 \mathrm{Da}$, $P 408$ 's formula is $P(E O)_{132}-b-P(P O)_{50}-b-P(E O)_{132}$ with an average molecular mass of $14600 \mathrm{Da}$, and $\mathrm{P} 407$ (also known as Kolliphor P407 or Pluronic F127) has the structure $\mathrm{P}(\mathrm{EO})_{100} \mathrm{-b}-$ $\mathrm{P}(\mathrm{PO})_{65}-\mathrm{b}-\mathrm{P}(\mathrm{EO})_{100}$ with an average molecular mass of 12600 Da. ${ }^{11-15}$ Aqueous solutions of P407 at concentrations of ca $15 \%$ $\mathrm{w} / \mathrm{w}$ and above have the ability to transform reversibly from liquid to gel when the temperature is increased above ca 15-25 ${ }^{\circ} \mathrm{C}$. This gelation occurs due to a hierarchical process of desolvation of the PPO core, which in turn leads to micellization and subsequent packing of these aggregates to form a face centred cubic gel mesophase. ${ }^{5}$

P407 is considered to be safe and is contained in medicines approved by the FDA. ${ }^{16}$ It has gained particular interest in topical drug delivery to mucosal membranes such as the vagina,

School of Life and Medical Sciences, University of Hertfordshire, U.K.

Electronic Supplementary Information (ESI) available: [rheograms and tables detailing composition of formulations]. See DOI: 10.1039/x0xx00000x eye, or rectum, ${ }^{5}$ where the materials may pass through an applicator before transforming into a retentive gel state upon contact with the body. ${ }^{5}$ However, there are some drawbacks with P407 in topical drug delivery, such as the weak gel strength, rapid dissolution in physiological fluids, and low forces of mucoadhesion. ${ }^{17-19}$ Alternative materials are reported in the literature, such as block copolymers consisting of poly(ethylene glycol) and poly( $\mathrm{N}$-isopropylacrylamide) which exhibit thermoreversible gelation. ${ }^{12}$ However, any novel materials would require a lengthy and costly toxicity testing programme before use in pharmaceuticals. Soluplus, a poly $(\mathrm{N}$-vinyl caprolactam) - poly(vinyl acetate) - poly(ethylene glycol) copolymer, is another alternative temperature-responsive polymer which is known to be safe and is approved for use in medicines. ${ }^{20}$ Soluplus solutions increase in viscosity when warmed, however, Soluplus requires high concentrations (30\% $\mathrm{w} / \mathrm{v}$ ) to thicken and has weak mechanical properties, forming thickened fluids rather than elastic gels. ${ }^{20}$ Thus, an ideal thermoresponsive polymer system would be have a $\mathrm{P} 407$ base that can be tuned using simple polymer additives for the required characteristics.

The strength of P407 gelators has been modulated using two main approaches: chemical modification of P407 to promote inter-micelle interactions, or by the addition of hydrophilic polymers, ${ }^{6}$ such as $\mathrm{P} 188,{ }^{21-23}$ poly(vinyl alcohol) (PVA), ${ }^{24}$ poly(ethylene glycol) (PEG), ${ }^{25}$ and cross-linked polyacrylic acids (PAAs), ${ }^{26-29}$ to the formulation to improve cohesion. Chemical modification has greatly improved gel strengths, with modified P407 thermoreversible gelators reported with elastic moduli $\left(G^{\prime}\right)$ up to $200 \mathrm{kPa}$ (where $20 \%(\mathrm{w} / \mathrm{w}$ ) P407 gels reach ca 10 $\mathrm{kPa}){ }^{30}$ However this approach requires time consuming, potentially costly, synthesis, purification and toxicity tests. The addition of hydrophilic polymers, on the other hand could cut the time and cost significantly, as well as allowing for the 
preparation of materials with modulated properties from existing pharmaceutical excipients. However, these studies have shown smaller improvements in $\mathrm{P} 407$ properties compared with chemical modification, often reducing gel strengths through a rheological antagonism ${ }^{6}$ and are typically limited to binary polymer mixtures using a trial and error approach, one variable at a time. ${ }^{31-37}$

Design-of-Experiments (DoE) approaches incorporate statistical analysis of experimental data to illustrate a comprehensive picture describing an established model and gives predictions depending on the results of relatively small number of experiments. ${ }^{38}$ Within the context of poloxamer-polymer mixtures, DoE could be used to achieve an optimum formulation, predict interactions, and identify problems using a significantly lower number of experiments compared to the one-variable-at-a-time approach. ${ }^{39,40}$ The DoE requires determination of the most significant factors that which influence the model's function, then performing experiments methodically as indicated by the design. ${ }^{38,41,42}$ Employing the DoE approach to rationally understand the effect of additives on the rheological properties of P407-based formulations can further pave the way for the identification of complex systems with improved properties. Individual effects may be extracted, giving information about the interaction between P407 and additives. Consequently, the aim of this work is to examine the optimisation of thermoreversible gelators using polymer blends containing P407 and a complex range of polymer additives. The complex formulations used in the DoE will be conducted at two levels (concentrations) for each component: P407 at 15 and 20 $\% \mathrm{w} / \mathrm{w}$, as $15 \% \mathrm{w} / \mathrm{w}$ is fractionally above the critical gelling concentration and $20 \% \mathrm{w} / \mathrm{w}$ is the most common concentration used in the literature, ${ }^{43-47}$ with P188, PVA, PEG, and PAA, all at $0.0-0.1 \% \mathrm{w} / \mathrm{w}$ guided by regular two-level (L16) DoE, where these relatively low concentrations are typical in related studies. ${ }^{6}$ These additives are selected as they are all hydrophilic polymers, have a history of use in pharmaceuticals, and represent a group of macromolecules possessing $\mathrm{H}$-bond acceptors (e.g. P188 and PEG ether groups), donors (PVA alcohol moieties), and ionic species (carboxylic groups of PAA), to probe inter-molecular interactions. Furthermore molecular weight is explored at two levels where this factor may affect the ability of the additive to bridge micelles, the intrinsic viscosity of the polymer chains, and size of hydrated chains, for instance. ${ }^{48}$ The DoE approach will be used to understand the individual effects in complex polymer mixtures and to optimise gel strength of the thermoreversible gelators.

\section{Material and Methods}

\section{Materials}

P407, P188, L-PVA (Mw: 13,000-23,000 g/mol) 98\% hydrolysed, H-PVA $(85,000-124,000 \mathrm{~g} / \mathrm{mol}) 99 \%$ hydrolysed, L-PEG $(10,000$ $\mathrm{g} / \mathrm{mol}), \mathrm{H}-\mathrm{PEG}(100,000 \mathrm{~g} / \mathrm{mol})$, and L-PAA sodium salt solution $35 \% \mathrm{w} / \mathrm{v}$ in water $(15,000 \mathrm{~g} / \mathrm{mol})$ were purchased from SigmaAldrich (U.K.). H-PAA $25 \% \mathrm{w} / \mathrm{v}$ solution $(240,000 \mathrm{~g} / \mathrm{mol})$ was purchased from Acros Organic (U.K.). All reagents were used as supplied without further purification.

\section{Design of Experiment (DoE) approach to designing thermoreversible gelators from complex polymer mixtures}

A regular two-level DoE was implemented to statistically evaluate and develop a model for the evaluation and subsequent optimisation of octonary P407 formulations using the Design Expert $^{\circledR}$ (DE) software from Stat-Ease (USA). The independent variables (factors) that have been investigated were P407, P188, PVA, PEG and PVA, with the latter three additives at two molecular weights where the acronym " $\mathrm{L}$ " designates the lower molecular weight and " $\mathrm{H}$ " the higher molecular weight (vide supra).

Eight factors were identified for exploration. Thus, the L16 regular two-level factorial design has been chosen since it was deemed to provide an appropriate model resolution. The regular two-level model's resolution depends on the number of factors and experiments needed to build the model, with a full resolution requiring 2 (number of factors) experiments ( 256 for eight factors). The more experiments conducted, the higher the resolution. ${ }^{49,50}$ A resolution of 4 was selected, which is a compromise between resolution and the number of training formulations needed, which is $16 .^{51}$ The factors and levels were set at: P407 at two different levels (15 and $20 \% \mathrm{w} / \mathrm{w}$ ), P188 at two different levels ( 0 and $0.1 \% \mathrm{w} / \mathrm{w}$ ), PVA at two molecular weights $(\mathrm{L})$ and $(\mathrm{H})$ each at two levels $(0$ and $0.1 \% \mathrm{w} / \mathrm{w}), \mathrm{PEG}$ at two molecular weights $(\mathrm{L})$ and $(\mathrm{H})$ each at two levels ( 0 and $0.1 \%$ $\mathrm{w} / \mathrm{w})$, and PAA at two molecular weights $(\mathrm{L})$ and $(\mathrm{H})$ each at two levels ( 0 and $0.1 \% \mathrm{w} / \mathrm{w})$.

Sixteen training formulations were generated in DE to build a model (Table 1), each with four replicate experimental measurements. The responses for this design were the elastic modulus $\left(G^{\prime}\right)$, representing the gel strength, ${ }^{44}$ and gelation temperature (Tgel) aiming to investigate the effect of the polymer additives on $\mathrm{P} 407$ formulations. The 16 formulations were evaluated by rheometric temperature ramps to obtain $\mathrm{G}^{\prime}$ at $37{ }^{\circ} \mathrm{C}\left(\mathrm{G}^{\prime}{ }_{\left.37^{\circ} \mathrm{C}\right)}\right.$ and Tgel as Response 1 and Response 2, respectively. Pareto charts, which show the absolute values of 


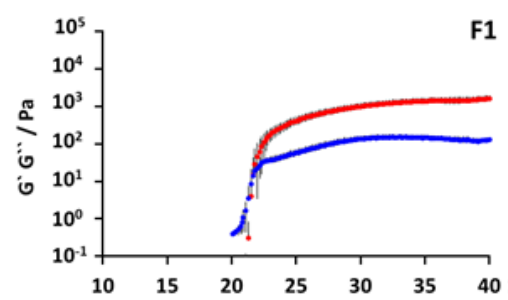

F1

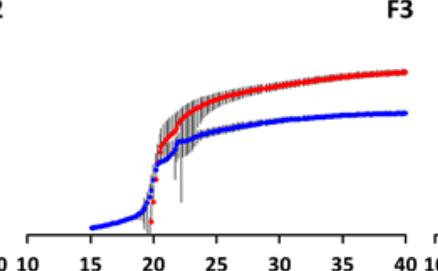

F3

F4

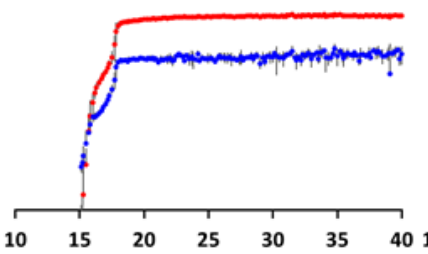

F6
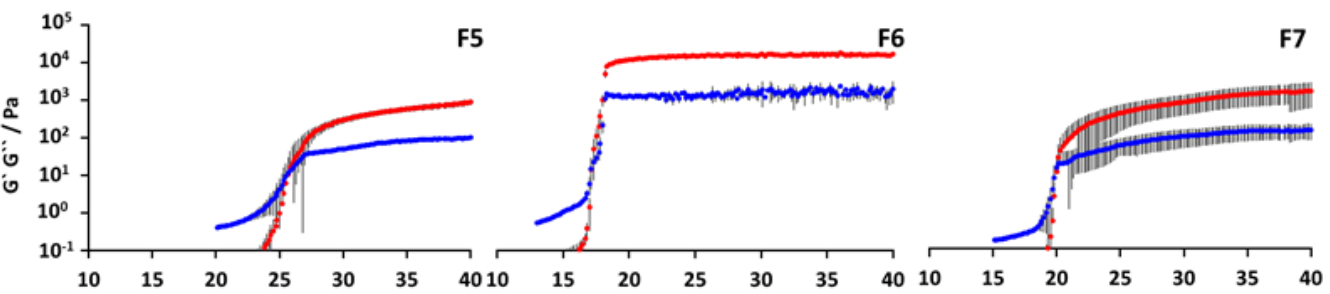

F7
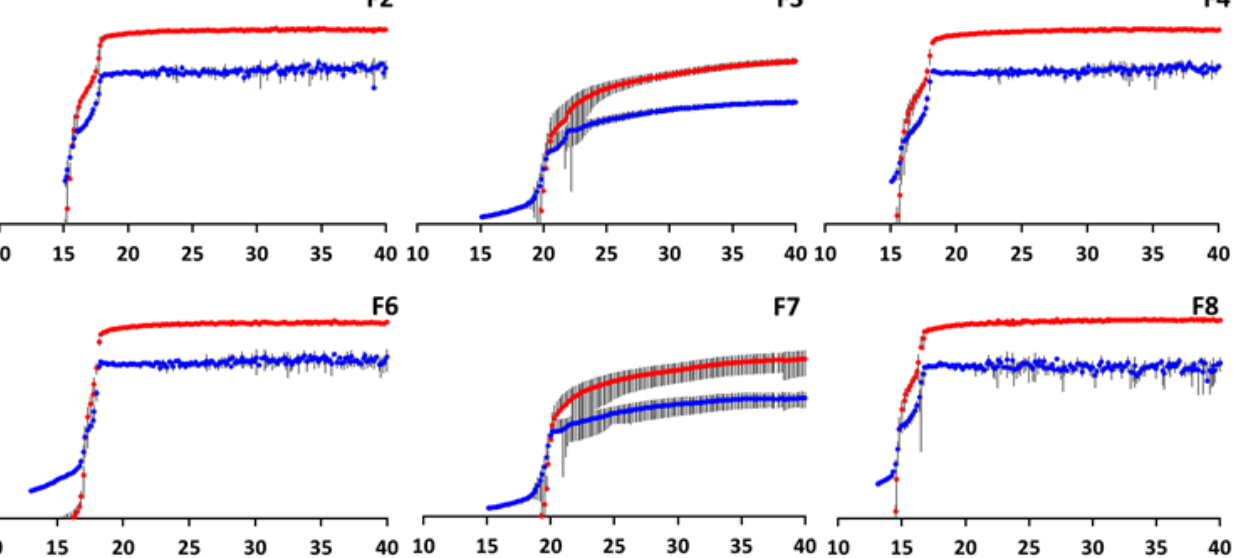

F10
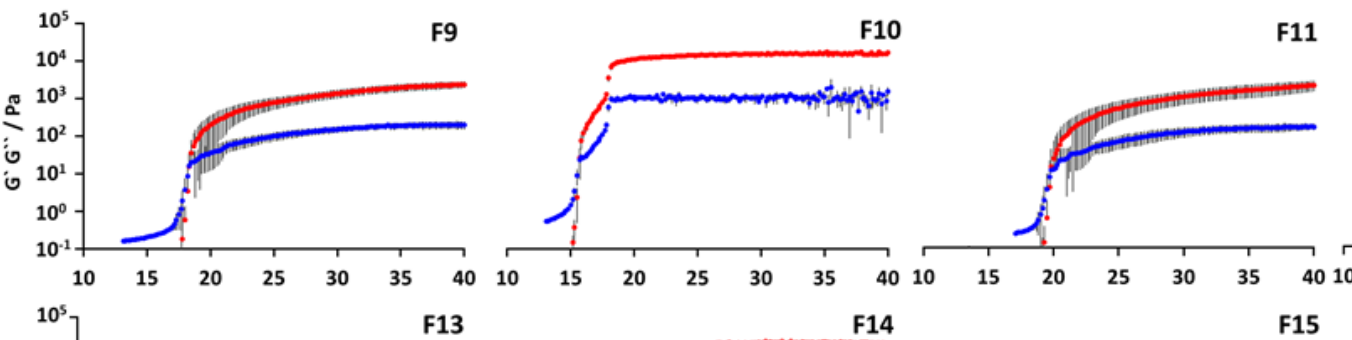

11

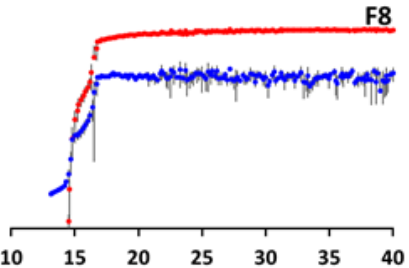

F8
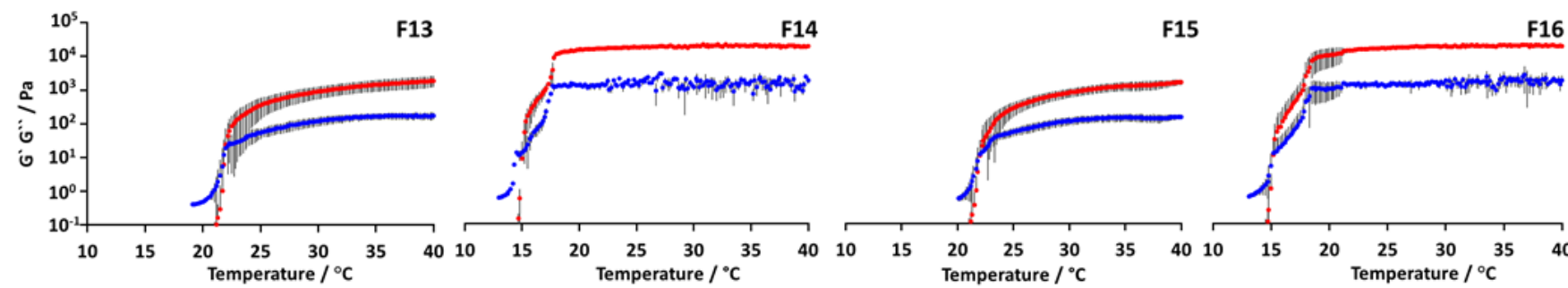

Figure 1: The DoE test formulation rheograms used to build the model ( $n=4, \pm S D$ ), where $\left(G^{\prime}\right)$ the storage and ( $\left.G^{\prime \prime}\right)$ loss modulus are illustrated in red and blue, respectively. Please refer to table 1 for the composition of these formulations.

the effects in a rank-order manner, were obtained for $\mathrm{G}^{\prime}$ and Tgel.

The developed model was then challenged by means of a subset of so-called "test" and "extended test" series, each containing five formulations, where the latter contains formulations with parameters outside of those used to generate the model. These were judiciously chosen as to maximise $\mathrm{G}^{\prime}{ }_{37^{\circ} \mathrm{C}}$ with $\mathrm{Tgel}$ greater than $15.8{ }^{\circ} \mathrm{C}$, which is the Tgel of P407 $20 \% \mathrm{w} / \mathrm{w}$ solution, to allow for a sol-gel transition to occur. Priority for the DoE solutions was given to $G^{\prime}$ maximisation, as samples could feasibly be kept in a cool environment to allow in situ gelation to occur when Tgel $=15^{\circ} \mathrm{C}$.

\section{Preparation of the polymer "training" formulations}

The training formulations (composition in Table 1) were prepared using the following steps: PEG $(\mathrm{L} / \mathrm{H})$ and PAA $(\mathrm{L} / \mathrm{H})$ were added to $10 \mathrm{~g}$ of phosphate buffer $(0.02 \mathrm{M})$ and stirred until complete dissolution. PVA (L,H) stock solutions of 4 (L) and $2(\mathrm{H}) \% \mathrm{w} / \mathrm{w}$, was prepared by heating the solution under the reflux for 10 minutes with stirring. The stock solutions were then diluted into the phosphate buffer/polymer mixtures to the required concentrations. P407 and P188 were then added and the formulation's weight was made to a total of $20 \mathrm{~g}$ with distilled water and placed in an ice-cold water with stirring for approximately $1 \mathrm{~h}$ until the polymers were completely dissolved.

\section{Temperature ramp rheometry of training and test formulations predicted by the DoE}

All rheology experiments were performed using a TA instruments AR 1500 ex rheometer fitted with a solvent trap using a parallel plate geometry ( $40 \mathrm{~mm}$ in diameter), a 600 $\mu \mathrm{m}$ gap, and an air pressure of 30 bar. All polymer solutions used for rheology were prepared as described previously and kept in the fridge at $4{ }^{\circ} \mathrm{C}$ overnight before analysis. The samples were left to equilibrate for $5 \mathrm{~min}$ at $10{ }^{\circ} \mathrm{C}$ on the rheometer peltier plate. Then temperature ramp measurements were carried out, with temperatures increased from $10{ }^{\circ} \mathrm{C}$ to $40{ }^{\circ} \mathrm{C}$ at a rate of $1{ }^{\circ} \mathrm{C} / \mathrm{min}$. This heating rate is relatively low to minimise lag in the sol-gel transition due to uneven sample warming. ${ }^{35,52,53}$ The measurements for all the formulations were carried out at a frequency of $1 \mathrm{~Hz}$ and oscillatory stress 
Table 1. Formulation composition and the $\mathrm{G}^{\prime}{ }_{37^{\circ} \mathrm{C}}$ and Tgel responses for the test formulations generated (mean $\left.\pm \mathrm{SD}\right)$

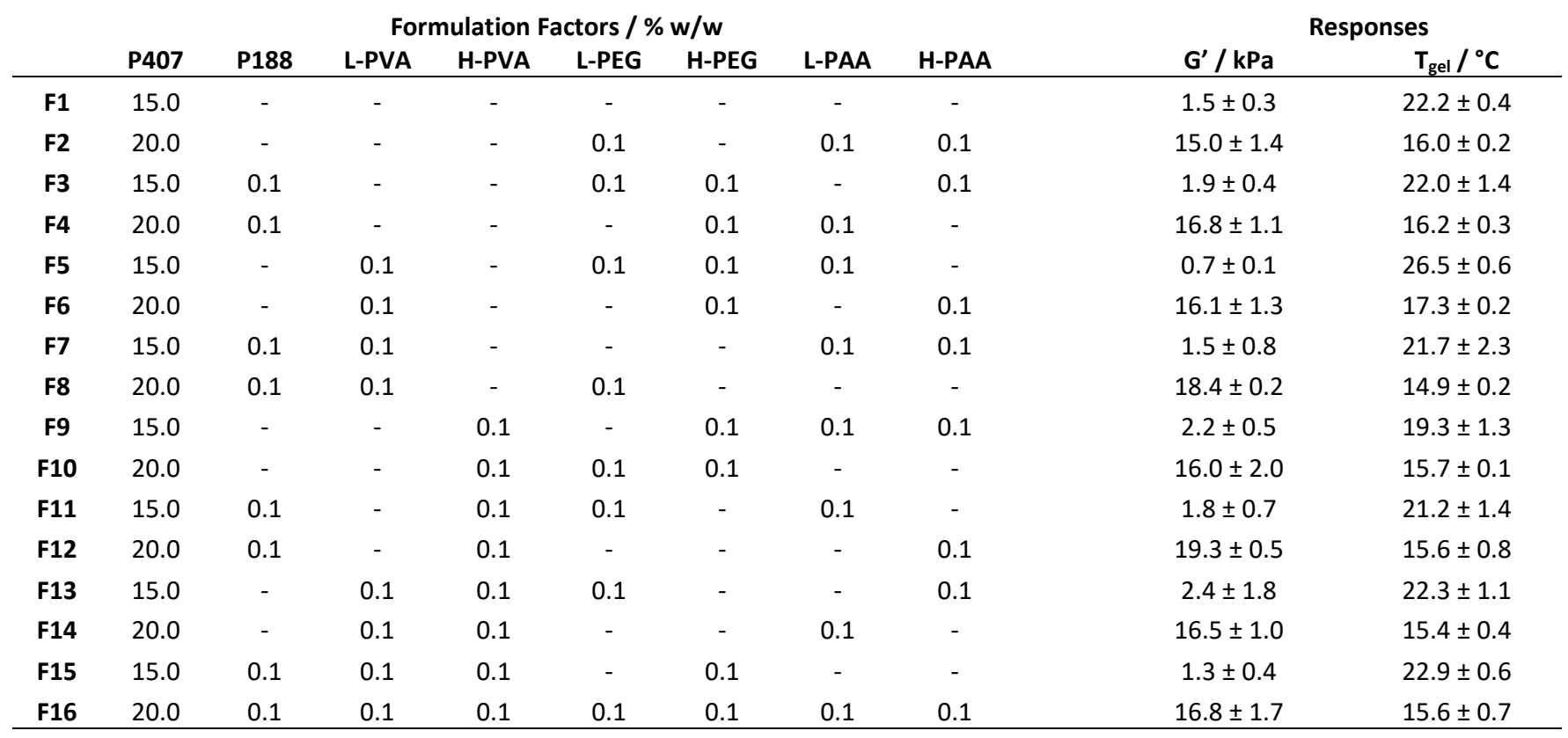

* formulations with $\mathrm{G}^{\prime}$ significantly higher than $\mathrm{G}^{\prime}$ for $\mathrm{P} 407$ at $37^{\circ} \mathrm{C}$. Statistical analysis conducted by one-way ANOVA with Tukey post-hoc analysis.

of $1 \mathrm{~Pa}$, which were found to be within the linear viscoelastic region of the $\mathrm{P} 40720 \% \mathrm{w} / \mathrm{w}$ solution.

\section{Results and discussion}

\section{The Design of Experiment (DoE) model for complex polymer mixtures}

Rheometric temperature ramps were conducted four times for each training set formulation to determine gel strength and Tgel, with data shown in Figure 1 for each training formulation. An exemplar formulation, F1, is shown in Figure $1 \mathrm{~A}$. In this sample, $\mathrm{G}^{\prime \prime}$ was greater than $\mathrm{G}^{\prime}$ at $20^{\circ} \mathrm{C}$, which indicates that the solution was behaving as a liquid-like material. When the sample reaches approximately $22{ }^{\circ} \mathrm{C}, \mathrm{G}$ ' becomes greater than $\mathrm{G}^{\prime \prime}$, indicating the dominance of elastic behaviour at this frequency. ${ }^{54}$ Across this transition, the absolute value of $\mathrm{G}^{\prime}$ increases from approximately $0.5 \mathrm{~Pa}$ at $20^{\circ} \mathrm{C}$ up to $1.5 \pm 0.3 \mathrm{kPa}$ at $37^{\circ} \mathrm{C}$, which was taken as the gel strength of this sample $\left(\mathrm{G}^{\prime}{ }_{37^{\circ} \mathrm{C}}\right)$. To exemplify the diversity of the rheograms in the dataset, Figure 1 shows a temperature ramp for all formulations. For example, F10 which has a $\mathrm{G}^{\prime}{ }_{37^{\circ} \mathrm{C}}$ of $16.0 \pm 2.0 \mathrm{kPa}$, approximately ten-fold greater than F1. On the other hand, Tgel was lower than F1, at $15.7 \pm 0.1{ }^{\circ} \mathrm{C}$. $\mathrm{G}^{\prime}{ }_{37^{\circ} \mathrm{C}}$ and Tgel were determined in this manner for the 16 experimental conditions.

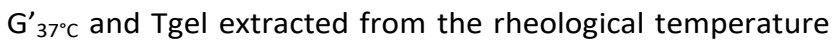
ramps for all 16 formulations is collected in Table 1 . From the resultant rheograms, it was clear that the formulations with P407 at $15 \% \mathrm{w} / \mathrm{w}$ (odd numbered formulations) had a significantly lower $\mathrm{G}^{\prime}{ }_{37^{\circ} \mathrm{C}}$ and higher Tgel comparing with the even-numbered formulations which had P407 at $20 \% \mathrm{w} / \mathrm{w}$. P407 at $20 \% \mathrm{w} / \mathrm{w}$ without additives is show in Figure S1.

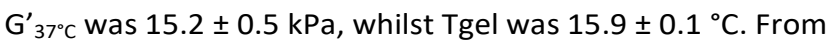
the rheology data in Table 1, a significant improvement ( $\mathrm{p}<$ 0.05 by one-way ANOVA with Tukey's test) in $\mathrm{G}^{\prime}{ }_{37^{\circ} \mathrm{C}}$ was observed for F8 and F12 compared with the P407 solution without additives.

\section{Investigation into individual effects within the DoE}

Pareto charts were generated which illustrate how individual factors affect $\mathrm{G}^{\prime}{ }_{37^{\circ} \mathrm{C}}$ and Tgel as predicted by the model. Positive and negative effects are assigned based on a positive or negative increase of the output variable, i.e. a positive effect indicates an increase in the absolute value $\mathrm{G}^{\prime}$ or Tgel, whilst a negative effect indicates a reduction. Equation 1 and 2 denote the coded factorial fitted models for Tgel and $\mathrm{G}^{\prime}{ }_{37^{\circ} \mathrm{C}}$, respectively.

Tgel $=+19.07-3.20 \cdot A-0.2844 \cdot B+0.5375 \cdot C-$ $0.5469 \cdot D+0.2313 \cdot E+0.4000 \cdot F-0.3344 \cdot H-0.5688$.

$A C+0.2844 \cdot A D-0.5125 \cdot A E+0.6094 \cdot A H$ $G^{\prime}{ }_{37^{\circ} \mathrm{C}}=+9305.18+7637.07 \cdot A+435.72 \cdot B-318.45 \cdot F-$ $326.74 \cdot G+462.03 \cdot A B$

In equation 1 and 2, each letter represents a factor (see Table 1 for details) and the coefficients indicate the relative impact of each factor on the evaluated rheological property. 
In turn, the coefficient denotes the average of all actual responses. Careful evaluation indicates that P407 exerts the largest impact of all factors, particularly in the case of the strength of the formulation. It is of note that Tgel is determined by a significantly larger number of factors, and combinations of them, when compared to the coded

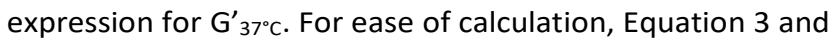
4 represent the predictive models whereby the factors are expressed in their original units.

$$
\begin{aligned}
& \text { Tgel } /{ }^{\circ} \mathrm{C}=+40.17187-1.20625 \cdot[P 407]-5.68750 \cdot \\
& {[P 188]+90.37500 \cdot[L-P V A]-50.75000 \cdot[H-P V A]+} \\
& 76.37500 \cdot[L-P E G]+8.00000 \cdot[H-P E G]-92.00000 \cdot \\
& {[H-P A A]-4.55000 \cdot[P 407] \cdot[L-P V A]+2.27500 \cdot} \\
& {[P 407] \cdot[H-P V A]-4.10000 \cdot[P 407] \cdot[L-P E G]+} \\
& 4.87500 \cdot[P 407] \cdot[H-P A A]
\end{aligned}
$$

$G_{37^{\circ} \mathrm{C}}^{\prime}=-40710.61875+2870.01500 \cdot[P 407]-$

$55970.25000 \cdot[P 188]-6368.90625 \cdot[H-P E G]-$

$6534.71875 \cdot[L-P A A]+3696.26250 \cdot[P 407] \cdot[P 188]$

From the Pareto charts shown in Figure 2, P407 has the most significant effect on the model whether relating to $\mathrm{G}^{\prime}{ }_{37^{\circ} \mathrm{C}}$ or Tgel. This is at least in part due to the greater concentration
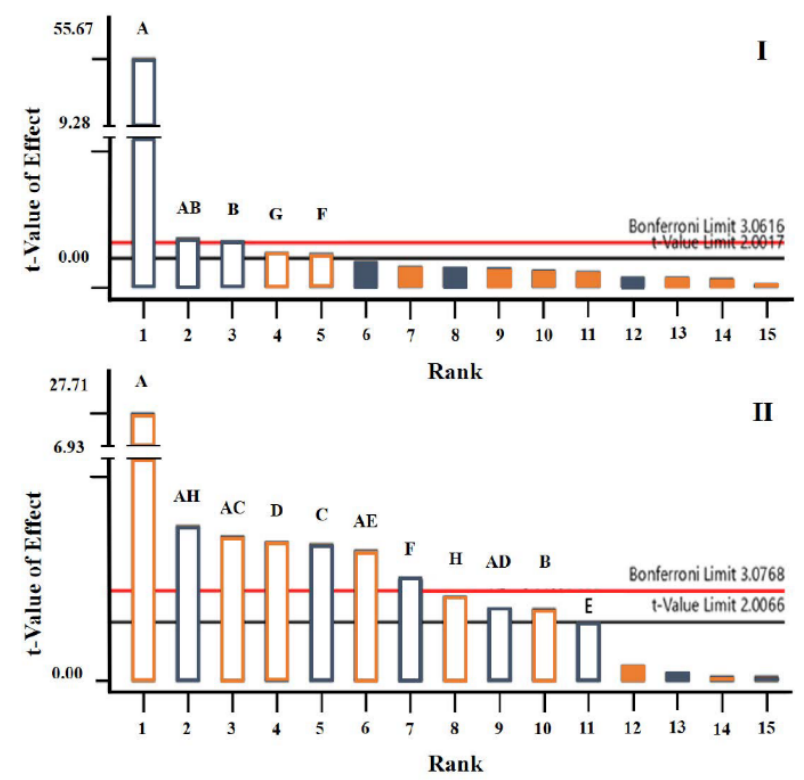

Figure 2. Pareto charts generated from the DoE model. (I) Pareto chart showing the positive and negative effects on $\mathrm{G}^{\prime} 37^{\circ} \mathrm{C}$ (II) Pareto chart showing the positive and negative effects on Tgel. Where A, B, C, D, E, F, G and $\mathrm{H}$ represent P407, P188, L-PVA, H$P V A, L-P E$, respectively. Positive effects were presented in grey colour and the negative effect presented in orange where the unfilled represent the selected effects and the filed ones are below $\mathrm{t}$-value limit and not been selected. difference between the two levels in P407. The pareto charts indicate that $\mathrm{P} 407$ has a positive effect on $\mathrm{G}^{\prime}{ }_{37^{\circ} \mathrm{C}}$ and negative effect on Tgel, consistent with the literature. ${ }^{55}$ From the pareto chart for $\mathrm{G}^{\prime}{ }_{37^{\circ} \mathrm{C}} \mathrm{P} 188$ has a significant positive effect on the model, which indicates that $\mathrm{G}^{\prime}{ }_{37^{\circ} \mathrm{C}}$ would increase with the increase of $\mathrm{P} 188$, and the two thus exhibit a rheological synergism. This is compatible with prior literature, where the formulation's viscosity increased with P188 in the formulation. For example Xuan et al. showed that the viscosity of a P407/P188 15/15\% w/w mixture was 151 $\mathrm{mPa}$.s which increased to $235 \mathrm{mPa}$.s for a P407/P188 15/20 \% formulation. ${ }^{56}$ This rheological synergism may be attributed to a mechanism proposed by Zhang et $\mathrm{al}^{57}$ whereby the two poloxamers do not form a mixed micellar system, but the low concentrations of P188 in the extramicellar space of the P407 liquid crystalline mesophase slows diffusion of the micelles during exposure to shear. P188 is expected to be in the unimer state in this study, where a critical micellization temperature is not exhibited within the temperature range studied. ${ }^{58}$ L-PAA and H-PEG had a significant negative effect, at 95\% confidence, on

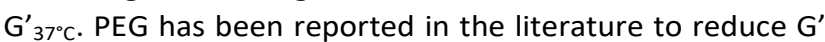
with the increase of PEG concentration, 25 and that higher molecular weights of PEG disrupt gel formation at lower concentrations. ${ }^{59}$ This has been attributed to entropic effects and increases in osmotic pressure disfavouring micelle formation, particularly for larger molecular weight polymers which are sterically excluded from the micelle. ${ }^{60}$ For L-PAA G' has reduced in another study from 16 to $14 \mathrm{kPa}$ when increasing concentration from 0.1 to $0.15 \% \mathrm{w} / \mathrm{w}$, respectively. ${ }^{29}$ Disruption of micelle formation is typically attributed as the causative factor for this observation.

Tgel was investigated by the model, and the pareto chart is shown in Figure 2II. A significant negative effect was seen for P407, where an inverse proportionality is expected between P407 concentration and Tgel as the critical phase volume of micelles for gelation to occur is reached at a lower temperature. ${ }^{61} \mathrm{H}-\mathrm{PVA}, \mathrm{H}-\mathrm{PAA}$ and $\mathrm{P} 188$ showed a significant negative effect on Tgel while L-PVA, H-PEG and L-PEG showed a significant positive effect. For P188 and PAA, the model was able to predict the formulation's Tgel behaviour in agreement with the literature. Carbopol and Polycarbophil, cross-linked PAAs, have been reported to supress Tgel ${ }^{29,35,62}$ as has P188. ${ }^{31}$ The model also concurred with findings in the literature for the positive effect of PEG on Tgel. ${ }^{60} \mathrm{~A}$ study by Edsman et al. found that increased concentration of PEG in a P407 formulation caused an increase in Tgel from 17.5 to $25.5^{\circ} \mathrm{C}$ when increasing PEG concentration from 2 to $5 \%$, respectively, in a $25 \% \mathrm{P} 407$ formulation. ${ }^{25}$ For L-PVA and H-PVA, we observe two different behaviours, when using a higher molecular weight $\mathrm{H}-\mathrm{PVA}$, the polymer has a negative effect on the Tgel which means the increase in H-PVA concentration would decrease Tgel, while L-PVA showed a positive effect on Tgel. The behaviour of H-PVA in our model is in accordance with a study by Bercea et al. which used PVA at a Mw of 76 kDa and 

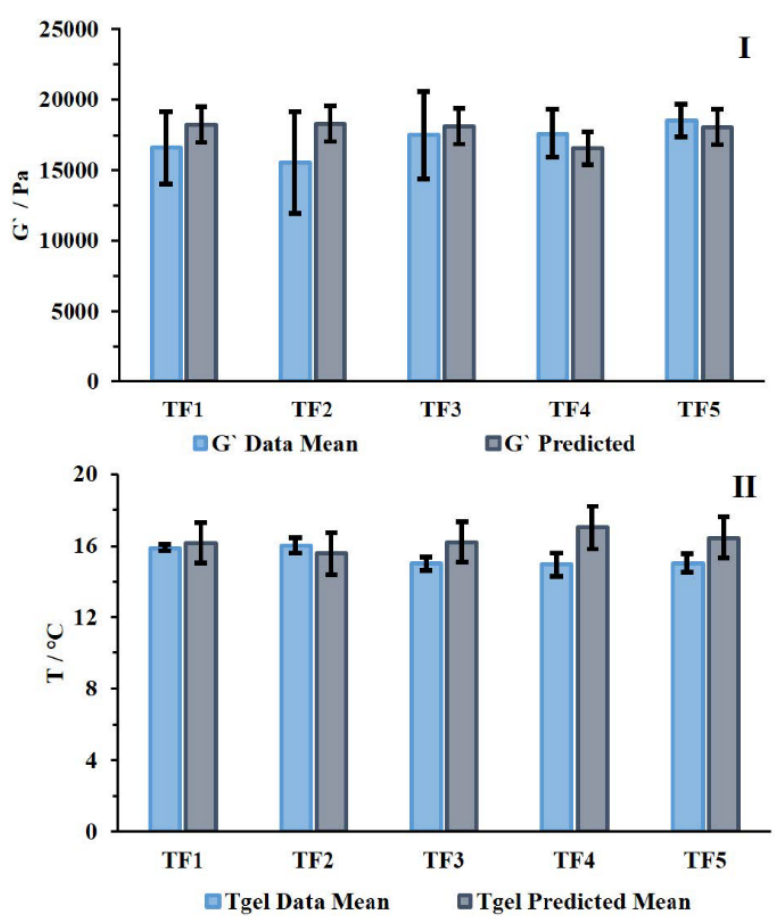

Figure 3: (I) the $\mathrm{G}^{\prime}$ measured data mean $\pm \mathrm{Cl}$ at $95 \%$ confidence (n =4) for five test formulations vs G' PI range for each formulation, (II) the Tgel measured data mean $\pm \mathrm{Cl}$ at $95 \%$ confidence $(\mathrm{n}=4)$ for five formulations vs Tgel PI range for each formulation. Where the error bars represent the $\mathrm{Cl}$ and $\mathrm{PI}$ range.

found a negative effect on $\mathrm{P} 407$ polymer blend Tgel. ${ }^{24}$ To our knowledge, L-PVA has not been reported in the literature as an additive to P407 formulations. Thus, this study illustrates the importance of additive molecular weight on thermogel properties, in addition to additive chemical functionality. Changes in Tgel are often attributed to the disruption of micelle formation caused by the additives, which lead to the increase of Tgel, or the increase of polymer concentration which leads to a reduction in Tgel through competition for solvent. An increase in $G^{\prime}$ would be attributed either to a promotion of inter-micelle interaction, or feasibly through the increased viscosity of extramicellar aqueous domains.

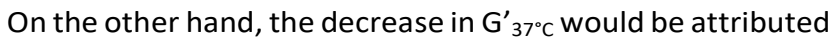
to disruption of micelle structure or packing behaviour. 6,63 Whilst a reduction in Tgel could intuitively lead to an

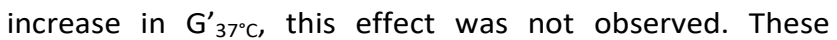
findings also highlight the complexity of optimising P407 formulations, where chemically similar additives may have vastly different effects, for example PVA elevating or reducing Tgel depending on molecular weight.

To rationalise molecular weight effects several different paradigms may be adopted. One hypothesis may be developed by considering inter-micelle interactions. It is hypothesised that the higher molecular weight PVA and PAA systems are capable of increasing cohesion between P407 micelles due to $\mathrm{H}$-bond donation and ion-dipole interaction between the hydroxyl and carboxylate groups, respectively, and the ether moieties of PEG chains in the P407 corona. This improved cohesion results in sufficient elasticity to induce gelation at a lower volume fraction of micelles, and thus temperature. This may be weighed against a consideration that the mesophase is a result of liquid crystallinity of the P407 micelles which may be negatively impacted by the presence of additive, and thus a positive effect on $\mathrm{G}^{\prime}{ }_{37^{\circ} \mathrm{C}}$ may not be seen. The lower molecular weight polymers may not be sufficiently large to bridge micelles. The effect of PEG is never positive where these cohesive intermolecular interactions would be relatively weak.

\section{Testing of the DoE model}

We challenged the model by generating predictions of new formulations, i.e. test formulations (TFs) external to those used to build the model. The chosen parameters of the TFs are consistent with those used to generate the model. To pave the way for the realisation of superior P407-based formulations, the optimal formulation parameters were set as to maximize both $\mathrm{G}^{\prime}{ }_{37^{\circ} \mathrm{C}}$ and Tgel. As such, lowest levels for both rheological parameters were set to $15.2 \mathrm{kPa}$ and $15.8{ }^{\circ} \mathrm{C}$, for $\mathrm{G}^{\prime}{ }_{37^{\circ} \mathrm{C}}$ and Tgel, respectively, which are the characteristic parameters for $20 \%$ (w/v) P407 thermogels in the absence of any additives. To evaluate the model, five TFs representing the highest predicted $\mathrm{G}^{\prime}{ }_{37^{\circ} \mathrm{C}}$ values (Table S1) were prepared and rheometry conducted to determine the $\mathrm{G}^{\prime}{ }_{37^{\circ} \mathrm{C}}$ and Tgel values and whether these fall within the prediction interval region.

$\mathrm{G}^{\prime}{ }_{37^{\circ} \mathrm{C}}$ and Tgel for the five TFs that been predicted by the model are shown in Figure 3. The resulted G' falls within the predicted interval (PI) in all cases. While all the Tgels fall within the PI range except formulation TF4 which fell slightly below. This would indicate that, within the studied parameters, the model was able to predict $\mathrm{G}^{\prime}{ }_{37^{\circ} \mathrm{C}}$ for the test formulations. For Tgel, the model was predictive for four of the five formulations.

Judicious analysis of our results, show a statistically significant improvement in the test formulations for $\mathrm{G}^{\prime}{ }_{37^{\circ} \mathrm{C}}$ over native $\mathrm{P} 407$ solutions in some cases. For example, formulation $\mathrm{F} 5$, has a $\mathrm{G}^{\prime}{ }_{37^{\circ} \mathrm{C}}$ of $18 \mathrm{kPa}$ which is $3 \mathrm{kPa}$ higher than $\mathrm{P} 40720 \% \mathrm{w} / \mathrm{w}$, equivalent to a $20 \%$ increase in gel strength as well as a $1 \mathrm{kPa}$ increase in $\mathrm{G}^{\prime}{ }_{37^{\circ} \mathrm{C}}$ compared with the highest $G^{\prime}$ obtained in the literature to our knowledge. ${ }^{29}$ Thus, we have demonstrated that the use of DoE has helped to provide a powerful insight into the polymer blends properties, which in turn led to the improvement of the rheology of polymer mixtures containing P407 as well as a model capable of predicting the rheological behaviour of thermoresponsive polymer mixtures.

The model's ability to predict responses with parameters extended outside of the range used to generate the model was then evaluated. The response parameter was modified to be maximised using $15-25 \% \mathrm{w} / \mathrm{w}$ for P407, $0-0.3 \%$ $\mathrm{w} / \mathrm{w}$ for the rest of the additives while keeping the response 


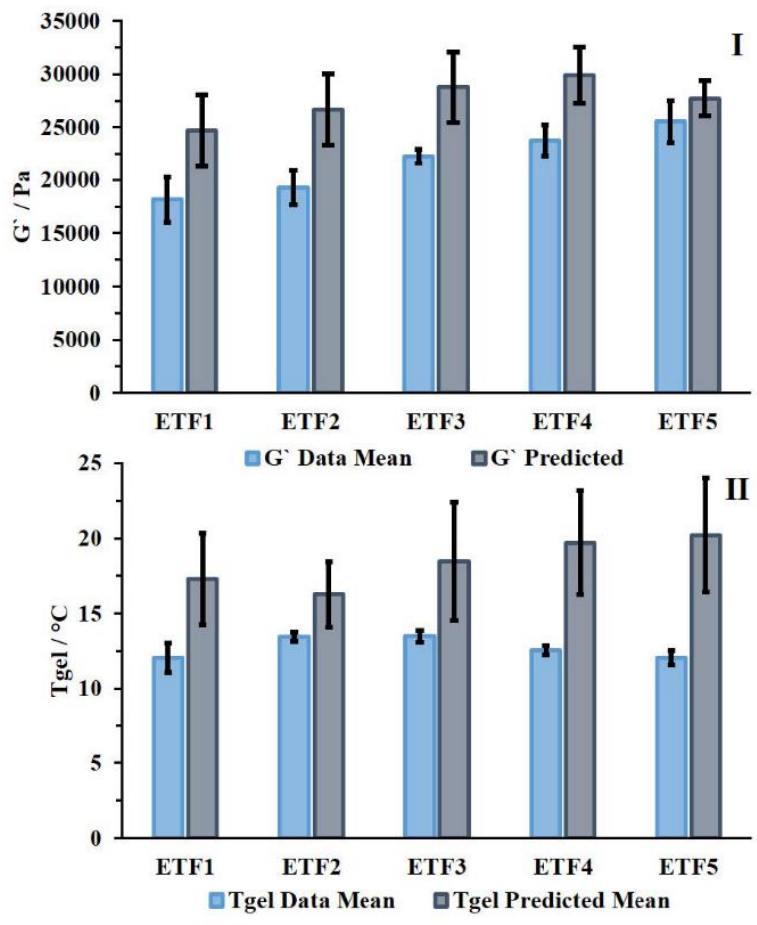

Figure $4:$ I) the $\mathrm{G}^{\prime}$ data mean $\pm \mathrm{Cl}$ at $95 \%$ confidence for five formulations at the extended parameters vs G' PI range for each formulation, II) the Tgel data mean $\pm \mathrm{Cl}$ at $95 \%$ confidence for five formulations at the extended parameters vs Tgel PI range for each formulation. Where represent the data means and represent the PI mean.

parameter the same (i.e., maximising $\mathrm{G}^{\prime}{ }_{37^{\circ} \mathrm{C}}$ and Tgel while keeping priority for $\left.G^{\prime}\right)$. Five extended test formulations (ETFs) with the highest $\mathrm{G}^{\prime}{ }_{37^{\circ} \mathrm{C}}$ and were chosen (Table S2) and evaluated by rheometry. After preparing the formulations,

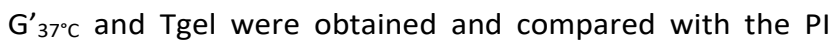
range. Within the extended tests formulations, ETF5's G' response and ETF2's Tgel response were within the PI range, as shown in Figure 4. Thus, the predictive ability of the model is strongest within the parameters used to generate it. Interestingly, some of the ETF formulations had a substantial improvement for $\mathrm{G}^{\prime}{ }_{37^{\circ} \mathrm{C}}$ (gel strength) over P407 $20 \% \mathrm{w} / \mathrm{w}$


large increase in $\mathrm{G}^{\prime}$ compared with P407 $20 \% \mathrm{w} / \mathrm{w}$ which is at $15 \mathrm{kPa}$. Although the Tgel responses were significantly lower than the PI range and lower than P407 $20 \% \mathrm{w} / \mathrm{w}$ (at $\sim 15{ }^{\circ} \mathrm{C}$ ), we have serendipitously generated a muchimproved polymer blend with properties which show promise for various applications such as drug delivery, bioprinting and tissue engineering. This effect may be attributed to the greater concentration of P407, however the gel strength of $25 \%(w / v)$ P407 is lower than the formulation, at ca $17 \mathrm{kPa}$, albeit in the absence of buffer. ${ }^{61}$

In summary, many studies have investigated the potential of supplementary additives to $\mathrm{P} 407$ formulations to improve its properties for various applications. ${ }^{6}$ For example, a formulation of P407 and methylcellulose showed an increase in gel viscosity at $37^{\circ} \mathrm{C},{ }^{64}$ where the addition of chitosan gave an improvement in the formulation's mucoadhesion properties as well as higher $G^{\prime}$ values. ${ }^{37}$ To get a comprehensive understanding of the effects of an additive using these one-variable-at-a-time approaches a large number of formulations are required. For instance, a study by Jones et al. ${ }^{35}$ required nineteen formulations containing different concentrations of P407 and carbopol to give an investigation of the effect of carbopol on P407 formulations. In our study, only sixteen formulations were used to investigate the addition of four different polymers with variation of molecular weight, totalling 8 factors. Using the DoE method allows an understanding of the critical effect of additives on the gel strength and Tgel of P407 formulations, and a predictive ability within the parameters used to generate the model. Although prior studies have used DoE approaches to explore P407, they were limited to a smaller number of additives and focused only on Tgel. ${ }^{39,40}$ Our study demonstrates efficacy in complex mixtures, allowing for prediction of novel and bespoke thermoresponsive gelators. This highlights DoE as a powerful tool to develop thermoresponsive formulations with complex composition appropriate for pharmaceutical dosage forms, bio-inks, and tissue engineering scaffolds, amongst other applications.

\section{Conclusions}

In this study, a DoE model has been designed to drive the development of thermoreversible P407 formulations using polymer additives in complex mixtures. The advantages of this method were the ability to predict an improved gel strength using only 16 training complex formulations to build up the model. Guided by an L16 regular two-level DoE, P407 formulation's gel strength was improved up to a maximum of $18 \mathrm{kPa}$ within the test formulations, and up to $25 \mathrm{kPa}$ using an extended test, i.e. 20 and $60 \%$ increase in gel strength, respectively, compared to a $20 \% \mathrm{w} / \mathrm{w} \mathrm{P} 407$ formulation. The model successfully predicted the responses when setting the polymer levels within the examined parameters used to build the model. This opens exciting avenues for optimisation of the parameters further to truly tailor these formulations. For example, larger statistical arrays containing all excipients approved for a specific route of administration may be conducted to develop the single most optimal gelators for a given medicine. Thus, the use of DoE in generating improved "smart" materials by identifying specific formulations with enhanced gel strength shows great promise. This approach would greatly help future researchers to optimise and understand complex thermoreversible formulations for a wide variety of healthcare and biomedical applications.

\section{Conflicts of interest}

There are no conflicts to declare. 


\section{Acknowledgements}

The University of Hertfordshire is thanked for supporting the research of MAS.

\section{References}

1 H. H. Lin and Y. L. Cheng, Macromolecules, 2001, 34, 37103715.

2 M. Teodorescu, I. Negru, P. O. Stanescu, C. Drghici, A. Lungu and A. Sârbu, React. Funct. Polym., 2010, 70, 790-797.

3 W. A. Petka, J. L. Harden, K. P. McGrath, D. Wirtz and D. A. Tirrell, Science (80-. )., 1998, 281, 389-392.

4 M. Hamidi, A. Azadi and P. Rafiei, Adv. Drug Deliv. Rev., 2008, 60, 1638-1649.

5 M. T. Cook and M. B. Brown, J. Control. Release, 2018, 270, 145-157.

6 M. A. Abou-Shamat, J. Calvo-Castro, J. L. Stair and M. T. Cook, Macromol. Chem. Phys., 2019, 1900173.

7 M. Sponchioni, U. Capasso Palmiero and D. Moscatelli, Mater. Sci. Eng. C, 2019, 102, 589-605.

8 R. Suntornnond, J. An and C. K. Chua, Macromol. Mater. Eng., 2017, 302, 1-15.

9 E. V. Batrakova and A. V. Kabanov, J. Control. Release, 2008, 130, 98-106.

10 M. S. H. Akash and K. Rehman, J. Control. Release, 2015, 209, 120-138.

11 J. B. da Silva, V. V. Khutoryanskiy, M. L. Bruschi and M. T. Cook, Int. J. Pharm., 2017, 528, 586-594.

12 C. Englert, J. C. Brendel, T. C. Majdanski, T. Yildirim, S. Schubert, M. Gottschaldt, N. Windhab and U. S. Schubert, Prog. Polym. Sci., 2018, 87, 107-164.

13 G. Wanka, H. Hoffmann and W. Ulbricht, Macromolecules, 1994, 27, 4145-4159.

14 M. R. Matanović, J. Kristl and P. A. Grabnar, Int. J. Pharm., 2014, 472, 262-275.

15 A. K. A. S. Brun-Graeppi, C. Richard, M. Bessodes, D. Scherman and O. W. Merten, Prog. Polym. Sci., 2010, 35, 1311-1324.

16 D. A. Gyles, L. D. Castro, J. O. C. Silva and R. M. RibeiroCosta, Eur. Polym. J., 2017, 88, 373-392.

17 G. Niu, F. Du, L. Song, H. Zhang, J. Yang, H. Cao, Y. Zheng, Z. Yang, G. Wang, H. Yang and S. Zhu, J. Control. Release, 2009, $138,49-56$

18 T. Gratieri, G. M. Gelfuso, O. De Freitas, E. M. Rocha and R. F. V. Lopez, Eur. J. Pharm. Biopharm., 2011, 79, 320-327.

19 E. S. A. Ibrahim, S. Ismail, G. Fetih, O. Shaaban, K. Hassanein and N. H. Abdellah, Acta Pharm., 2012, 62, 59-70.

20 I. Salah, M. Abou-Shamat and M. T. Cook, J. Appl. Polym. Sci., 2019, 136, 46915.

21 Z. M.A. Fathalla, A. Vangala, M. Longman, K. A. Khaled, A. K. Hussein, O. H. El-Garhy and R. G. Alany, Eur. J. Pharm. Biopharm., 2017, 114, 119-134.

22 J. Yun Chang, Y. K. Oh, H. Soo Kong, E. Jung Kim, D. Deuk Jang, K. Taek Nam and C. K. Kim, J. Control. Release, 2002, 82, 39-50.

23 S. Y. Karavana, S. Rençbe, Z. A. Şenyiğit and E. Baloğlu, Pharmacol. Pharm., 2012, 03, 417-426.

24 M. Bercea, R. N. Darie, L. E. Nita and S. Morariu, Ind. Eng. Chem. Res., 2011, 50, 4199-4206.

25 K. Edsman, J. Carlfors and R. Petersson, Eur. J. Pharm. Sci., 1998, 6, 105-112.

26 C. Karavasili and D. G. Fatouros, Drug Discov. Today, 2016, 21, 157-166.
27 S. B. De Souza Ferreira, T. D. Moco, F. B. Borghi-Pangoni, M. V. Junqueira and M. L. Bruschi, J. Mech. Behav. Biomed. Mater., 2017, 68, 265-275.

28 E. Altuntaş and G. Yener, AAPS PharmSciTech, 2017, 18, 2673-2682.

29 S. B. De Souza Ferreira, T. D. Moco, F. B. Borghi-Pangoni, M. V. Junqueira and M. L. Bruschi, J. Mech. Behav. Biomed. Mater., 2015, 55, 164-178.

30 D. Cohn, A. Sosnik and A. Levy, Biomaterials, 2003, 24 , 3707-3714.

31 E. Y. Kim, Z. G. Gao, J. S. Park, H. Li and K. Han, Int. J. Pharm., 2002, 233, 159-167.

32 R. J. Majithiya, P. K. Ghosh, M. L. Umrethia and R. S. R. Murthy, AAPS PharmSciTech, 2006, 7, 67.

33 Y. Liu, Y. ying Zhu, G. Wei and W. yue Lu, Eur. J. Pharm. Sci., 2009, 37, 306-312.

34 H. F. Salem, Int. J. Nanomedicine, 2010, 5, 943-954.

35 D. S. Jones, M. Luciano, O. De Freitas, M. Palmira, D. Gremião, E. Helena, G. Lara and G. P. Andrews, Int. J. Pharm., 2009, 372, 49-58.

36 M. H. M. Nascimento, M. K. K. D. Franco, F. Yokaichyia, E. de Paula, C. B. Lombello and D. R. de Araujo, Int. J. Biol. Macromol., 2018, 111, 1245-1254.

37 T. Gratieri, G. M. Gelfuso, E. M. Rocha, V. H. Sarmento, O. de Freitas and R. F. V. Lopez, Eur. J. Pharm. Biopharm., 2010, 75, 186-193.

38 E. Ban, D. J. Jang, S. J. Kim, M. Park and A. Kim, Pharm. Dev. Technol., 2017, 22, 939-945.

39 C. Pagano, S. Giovagnoli, L. Perioli, M. C. Tiralti and M. Ricci, Eur. J. Pharm. Sci., 2020, 142, 105125.

40 A. Khattab, S. Marzok and M. Ibrahim, J. Drug Deliv. Sci. Technol., 2019, 53, 101134.

41 L. Garcia-del Rio, P. Diaz-Rodriguez and M. Landin, Mater. Sci. Eng. C, 2020, 106, 110252.

42 S. Verma, S. K. Singh, P. R. P. Verma and M. N. Ahsan, Drug Dev. Ind. Pharm., 2014, 40, 1358-1370.

43 H. J. Chung, Y. Lee and T. G. Park, J. Control. Release, 2008, $127,22-30$.

44 S. H. Lee, Y. Lee, S. W. Lee, H. Y. Ji, J. H. Lee, D. S. Lee and T. G. Park, Acta Biomater., 2011, 7, 1468-1476.

45 G. Dumortier, J. L. Grossiord, F. Agnely and J. C. Chaumeil, Pharm. Res., 2006, 23, 2709-2728.

46 E. Ruel-Gariepy and J. C. Leroux, Eur. J. Pharm. Biopharm., 2004, 58, 409-426.

47 H. J. Moon, D. Y. Ko, M. H. Park, M. K. Joo and B. Jeong, Chem. Soc. Rev., 2012, 41, 4860-4883.

48 R. J. Young and P. A. Lovell, Introduction to Polymers, 1991. 49 P. Li, O. J. Akinlawon and S. Zhao, Commun. Stat. Simul. Comput., 2019, 0, 1-21.

50 Y. C. Hsiao, C. T. Liao and L. Y. D. Liu, Commun. Stat. Theory Methods, 2015, 44, 2764-2773.

51 P. Rafiei and A. Haddadi, Mater. Sci. Eng. C, 2019, 104, 111.

52 G. González-Gaitano, M. A. Da Silva, A. Radulescu and C. A. Dreiss, Langmuir, 2015, 31, 5645-5655.

53 J. B. da Silva, M. T. Cook and M. L. Bruschi, Carbohydr. Polym., , DOI:10.1016/j.carbpol.2020.116268.

54 L. Mayol, F. Quaglia, A. Borzacchiello, L. Ambrosio and M. I. La Rotonda, Eur. J. Pharm. Biopharm., 2008, 70, 199-206.

55 A. Fakhari, M. Corcoran and A. Schwarz, Heliyon, 2017, 3, e00390.

56 J. J. Xuan, P. Balakrishnan, D. H. Oh, W. H. Yeo, S. M. Park, C. S. Yong and H. G. Choi, Int. J. Pharm., 2010, 395, 317-323. 57 M. Zhang, M. Djabourov, C. Bourgaux and K. Bouchemal, Int. J. Pharm., 2013, 454, 599-610.

58 P. Alexandridis, J. F. Holzwarth and T. A. Hatton, Macromolecules, 1994, 27, 2414-2425. 
59 N. K. Pandit and R. Mcgowan, Drug Dev. Ind. Pharm., 1998,

24, 183-186.

60 M. Malmsten and B. Lindman, Macromolecules, 1993, 26, $1282-1286$

61 P. Haddow, W. J. McAuley, S. B. Kirton and M. T. Cook, 2020, Materials, Just Accepted.

62 E. Baloglu, S. Y. Karavana, Z. A. Senyigit, S. Hilmioglu-Polat, D. Y. Metin, O. Zekioglu, T. Guneri and D. S. Jones, J. Pharm. Pharmacol., 2011, 63, 1274-1282.

63 L. Mayol, M. Biondi, F. Quaglia, S. Fusco, A. Borzacchiello, L. Ambrosio and M. I. La Rotonda, Biomacromolecules, 2011 $12,28-33$.

64 Vigani, Faccendini, Rossi, Sandri, Bonferoni, Grisoli and Ferrari, Pharmaceutics, 2019, 11, 511. 